\title{
Ordered analytic Hilbert spaces over the unit disk
}

\author{
by \\ Shengzhao Hou and Shuyun Wei (Suzhou)
}

\begin{abstract}
Let $f, g$ be in the analytic function $\operatorname{ring} \operatorname{Hol}(\mathbb{D})$ over the unit disk $\mathbb{D}$. We say that $f \preceq g$ if there exist $M>0$ and $0<r<1$ such that $|f(z)| \leq M|g(z)|$ whenever $r<|z|<1$. Let $X$ be a Hilbert space contained in $\operatorname{Hol}(\mathbb{D})$. Then $X$ is called an ordered Hilbert space if $f \preceq g$ and $g \in X$ imply $f \in X$. In this note, we mainly study the connection between an ordered analytic Hilbert space and its reproducing kernel. We also consider when an invariant subspace of the whole space $X$ is similar to $X$.
\end{abstract}

1. Introduction. Let $\operatorname{Hol}(\Omega)$ denote the analytic function ring over the domain $\Omega \subset \mathbb{C}^{n}$. The most basic example of a Hilbert space contained in $\operatorname{Hol}(\Omega)$ is the famous Hardy space. The operator theory on the Hardy space has played an important role both in complex analysis and in functional analysis. Motivated by the results obtained in the Hardy space, the study of Hilbert spaces contained in $\operatorname{Hol}(\Omega)$ has recently drawn much attention. Among those Hilbert spaces, the Hardy space and Bergman space are very active fields of research, and many deep and interesting results have been obtained [CG, DP, HKZ2].

The most useful tool in the study of the Hardy space and Bergman space is the integration which induces the inner product. A difficulty arises when one deals with a Hilbert space whose inner product is not induced by a positive measure. In order to overcome it, the concept of ordered analytic Hilbert space over the complex plane has been introduced and has proved useful [CGH].

We note that both the Hardy space $H^{2}$ and the Bergman space $L_{\mathrm{a}}^{2}(\mathbb{D})$ have the following properties:

(1) the polynomial $\operatorname{ring} \mathcal{C}$ is dense in $X$;

(2) $X$ has a reproducing kernel;

(3) $\left\{z^{n}\right\}_{n=0}^{\infty}$ is an orthogonal basis of $X$ and $\liminf \sqrt[n]{\left\|z^{n}\right\|}=1$.

2000 Mathematics Subject Classification: 46E22, 47B32.

Key words and phrases: analytic Hilbert space, ordered analytic Hilbert space.

The authors are partially supported by NNSFC in China, No. 10301019, and Jiangsu Natural Science Foundation, No. BK2007049. 
Furthermore, if $f, g \in \operatorname{Hol}(\mathbb{D})$ and $|f(z)| \leq|g(z)|$ whenever $0<r<|z|<1$, then $g \in H^{2}$ (resp. $\left.g \in L_{\mathrm{a}}^{2}(\mathbb{D})\right)$ implies $f \in H^{2}$ (resp. $\left.f \in L_{\mathrm{a}}^{2}(\mathbb{D})\right)$. In the present paper, we are mainly concerned with Hilbert spaces contained in $\operatorname{Hol}(\mathbb{D})$ and having such properties.

In Section 2, we introduce an order structure in $\operatorname{Hol}(\mathbb{D})$. We give a necessary and sufficient condition for a Hilbert space to be ordered. The connection between the reproducing kernel and the "order structure" are discussed in Section 3; we give a criterion (Theorem 3.1) for a space to be ordered. Examples are given to show the necessity of each condition of Theorem 3.1. The similarity of subspaces to the whole space $X$ is studied in Section 4 and we give a complete description of such spaces. As an application of the "order structure", we prove that all finite-codimensional subspaces of an ordered analytic Hilbert space $X$ are similar to $X$, while this is not always the case when $X$ is not ordered.

We thank Professor Shunhua Sun for some helpful suggestions. We are deeply indebted to the referees for numerous suggestions that helped make this paper more readable.

2. Some basic properties of reproducing Hilbert space. In this section, we are concerned with the properties of analytic Hilbert spaces which will be used later.

Let $X$ be a Hilbert space consisting of analytic functions on the unit disk $\mathbb{D}$. Recall that $\lambda \in \mathbb{C}$ is a virtual point of $X$ if the homomorphism $p \mapsto p(\lambda)$ defined on the polynomial ring $\mathcal{C}$ extends to a bounded linear functional on $X$.

We begin with a definition which comes from [CG].

Definition 2.1. We say that $X$ is an analytic Hilbert space over $\mathbb{D}$ if the following conditions are satisfied:

(1) the polynomial $\operatorname{ring} \mathcal{C}$ is dense in $X$;

(2) $X$ has a reproducing kernel;

(3) the set of virtual points of $X$ is equal to $\mathbb{D}$.

We note that (3) guarantees that the reproducing kernels $K(z, \lambda)$ are precisely analytic in $z$ and conjugate analytic in $\lambda$ over $\mathbb{D}$. The main reason for requiring the virtual set to be open is that one can do calculations on it, in particular, analytic calculations. For example, one can do asymptotic analysis of the reproducing kernel on the boundary of the virtual set.

In both the Hardy and Bergman spaces, we know that $\lim \sqrt[n]{\left\|z^{n}\right\|}$ $=1$. In a general analytic Hilbert space $X$, under the condition that $\left\{z^{n}\right\}_{n=0}^{\infty}$ is an orthogonal basis of $X$, we have the following proposition which will be used several times in this paper. 
Proposition 2.1. Let $X$ be an analytic Hilbert space with orthogonal basis $\left\{z^{n}\right\}_{n=0}^{\infty}$. Then

$$
\liminf _{n \rightarrow \infty} \sqrt[n]{\left\|z^{n}\right\|}=1
$$

Proof. Let $K_{\lambda}$ be the reproducing kernel of $X$. We first show that

$$
\lim _{|\lambda| \rightarrow 1}\left\|K_{\lambda}\right\|=\infty
$$

In fact, if $\lim _{|\lambda| \rightarrow 1}\left\|K_{\lambda}\right\| \neq \infty$, then there exist $\lambda_{n}$ with $\left|\lambda_{n}\right|<1$ such that $\lim _{n \rightarrow \infty} \lambda_{n}=\lambda_{0}$ with $\left|\lambda_{0}\right|=1$ and

$$
\lim _{n \rightarrow \infty}\left\|K_{\lambda_{n}}\right\|=R<\infty .
$$

Since

$$
\left|p\left(\lambda_{n}\right)\right|=\left|\left\langle p, K_{\lambda_{n}}\right\rangle\right| \leq\|p\|\left\|K_{\lambda_{n}}\right\|,
$$

letting $n \rightarrow \infty$, we have $\left|p\left(\lambda_{0}\right)\right| \leq\|p\| R$. This contradicts the assumption that no $\lambda_{0}$ with $\left|\lambda_{0}\right|=1$ is a virtual point of $X$. Thus we have $\lim _{|\lambda| \rightarrow 1}\left\|K_{\lambda}\right\|=\infty$.

Now we are ready to show

$$
\liminf _{n \rightarrow \infty} \sqrt[n]{\left\|z^{n}\right\|} \leq 1
$$

Indeed, otherwise there exist $N \in \mathbb{N}$ and $\delta>0$ such that $\sqrt[n]{\left\|z^{n}\right\|}>1+\delta$ whenever $n>N$. Thus

$$
\begin{aligned}
\left\|K_{\lambda}(z)\right\|^{2} & =\left\|\sum_{m=0}^{\infty} \frac{\bar{\lambda}^{m} z^{m}}{\left\|z^{m}\right\|^{2}}\right\|^{2}=\sum_{m=0}^{\infty} \frac{|\lambda|^{2 m}}{\left\|z^{m}\right\|^{2}} \\
& \leq \sum_{m=0}^{N} \frac{|\lambda|^{2 m}}{\left\|z^{m}\right\|^{2}}+\sum_{m=N+1}^{\infty} \frac{|\lambda|^{2 m}}{(1+\delta)^{2 m}}<\infty,
\end{aligned}
$$

contrary to $\lim _{|\lambda| \rightarrow 1}\left\|K_{\lambda}\right\|=\infty$.

Next we will show that

$$
\liminf _{n \rightarrow \infty} \sqrt[n]{\left\|z^{n}\right\|} \geq 1
$$

Indeed, otherwise one can find a subsequence $n_{1}<n_{2}<\cdots$ and $0<r<1$ such that $\sqrt[n_{k}]{\left\|z^{n_{k}}\right\|}<r$ or equivalently $\left\|z^{n_{k}}\right\| \leq r^{n_{k}}$ for $k=1,2, \ldots$.

Choose $1>\delta>r$. Since $X$ is a reproducing Hilbert space, there exists $C_{\delta}>0$ such that $|f(\delta)| \leq C_{\delta}\|f\|$ for all $f \in X$. This gives

$$
\delta^{n} \leq C_{\delta}\left\|z^{n}\right\| .
$$

Thus

$$
\delta \leq C_{\delta}^{1 / n_{k}}\left\|z^{n_{k}}\right\|^{1 / n_{k}} .
$$

Now letting $k \rightarrow \infty$, we get $\delta \leq r$, which is not the case.

We note that there is a natural partial order on the ring $\operatorname{Hol}(\mathbb{D})$. Let $f, g$ be in $\operatorname{Hol}(\mathbb{D})$. We say that $f \preceq g$ if there exist $M>0$ and $0<r<1$ 
such that $|f(z)| \leq M|g(z)|$ whenever $r<|z|<1$. The next proposition gives a characterization of this partial order.

Proposition 2.2. Let $f, g$ be in $\operatorname{Hol}(\mathbb{D})$ and let $H^{\infty}$ denote the set of bounded analytic functions in $\mathbb{D}$. Then $f \preceq g$ if and only if $f / g=b / p$, where $b \in H^{\infty}$ and $p$ is a polynomial with all zeros in $\mathbb{D}$.

Proof. " $\Rightarrow$ " Without loss of generality, we may assume that $f$ and $g$ have no common zeros. If $f \preceq g$, then by definition there exist $0<r<1$ and $M>0$ such that $|f(z) / g(z)| \leq M$ whenever $r<|z|<1$. Therefore $g(z) \neq 0$ for $r<|z|<1$. Thus the zeros of $g$ in $\mathbb{D}$ are in $|z| \leq r$ and hence are finitely many, say $\alpha_{1}, \ldots, \alpha_{k}$. Let $p=\left(z-\alpha_{1}\right) \cdots\left(z-\alpha_{k}\right)$. Then $(f / g) p=: b$ is analytic and bounded in $\mathbb{D}$. Thus $b \in H^{\infty}$ and $f / g=b / p$.

" $\Leftarrow "$ If $f / g=b / p$, then $f=(b / p) g$. Since the zeros of $p$ are in $\mathbb{D}$, one can find $r>0$ such that $|p(z)| \geq m>0$ whenever $r \leq|z| \leq 1$. This together with the fact that $b \in H^{\infty}$ gives

$$
|f(z)| \leq \frac{M}{m}|g(z)| \quad \text { for } r<|z|<1 .
$$

This means that $f \preceq g$.

We say that an analytic Hilbert space $X$ is ordered if for $f, g \in \operatorname{Hol}(\mathbb{D})$, $f \preceq g$ and $g \in X$ imply $f \in X$. By definition, both the Hardy and Bergman spaces are ordered. The following proposition gives a description of such spaces and will be used several times in this paper.

Proposition 2.3. An analytic Hilbert space $X$ is ordered if and only if the following hold:

(1) for each $b \in H^{\infty}, f \in X$ implies bf $\in X$;

(2) for each $f \in X$ and each polynomial $p$ with all zeros in $\mathbb{D}, p f \in X$ implies $f \in X$.

Proof. Assume that $X$ is ordered. If $b \in H^{\infty}$ then there exists $M>0$ such that $|b f| \leq M|f|$. That is, $b f \preceq f$. So $f \in X$ implies $b f \in X$.

If $p$ is a polynomial with all zeros in $\mathbb{D}$, then there exist $r, m>0$ such that $|p(z)| \geq m$ whenever $r \leq|z| \leq 1$. Thus $|p(z) f(z)| \geq m|f(z)|$ whenever $r \leq|z| \leq 1$. So $f \preceq p f$. Thus $p f \in X$ implies $f \in X$.

If $f \preceq g$, we will prove that $g \in X$ implies $f \in X$ under the conditions (1) and (2). By Proposition 1.2, if $f \preceq g$, then $g=(p / b) f$. This together with the fact that $g \in X$ and (1) gives $b \cdot(p / b) f=p f \in X$. Now using (2) we have $f \in X$. This completes the proof.

Proposition 2.3 has the following equivalent and useful form.

Proposition 2.3'. An analytic Hilbert space $X$ is ordered if and only if the following hold: 
(1) for each $b \in H^{\infty}, f \in X$ implies bf $\in X$;

(2) for each $f \in X$ and $|\alpha|<1,(z+\alpha) f \in X$ implies $f \in X$.

Throughout this paper, we will consider analytic Hilbert spaces with orthogonal basis $\left\{z^{n}\right\}_{n=0}^{\infty}$; for brevity, we will call them just analytic Hilbert spaces.

Before continuing, we give two examples which show the existence of analytic Hilbert spaces without the desired order.

ExAMPLE 2.1. Let $X$ be an analytic Hilbert space with $\|1\|=1,\left\|z^{2 k}\right\|^{2}$ $=2 k$ and $\left\|z^{2 k+1}\right\|^{2}=1 /(2 k+1)$ for $k=1,2, \ldots$ Then $X$ is not ordered.

In fact, let $f(z)=\sum_{k=1}^{\infty} z^{2 k} / \sqrt{2 k}$. Then $\|f\|^{2}=\sum_{k=1}^{\infty}\left\|z^{2 k}\right\|^{2} / 2 k=\infty$. So $f \notin X$. But

$$
\|z f(z)\|^{2}=\sum_{k=1}^{\infty} \frac{z^{2 k+1}}{2 k}=\sum_{k=1}^{\infty} \frac{1}{2 k(2 k+1)}<\infty .
$$

This means that $z f \in X$. Now by Proposition 2.3, $X$ is not ordered.

Example 2.1 does not satisfy condition (2) of Proposition 2.3, while the following one does not satisfy condition (1):

ExAMPLE 2.2. Let $X$ be an analytic Hilbert space with $\left\|z^{2 k}\right\|^{2}=1 / 2 k$ and $\left\|z^{2 k+1}\right\|^{2}=2 k+1$. Then $X$ is not ordered.

In fact, let $f(z)=\sum_{k=1}^{\infty} z^{2 k} / \sqrt{2 k}$ and $b(z)=z$. Then it is easy to check $f \in X$ but $b f \notin X$. So $X$ is not ordered.

By the definition of an ordered analytic Hilbert space, it is easy to see that each analytic Hilbert space with inner product induced by a positive measure is ordered. The following proposition gives a necessary condition for having such a measure.

Proposition 2.4. Let $X$ be an analytic Hilbert space whose inner product is induced by a positive measure $\sigma$. Then $\lim _{n \rightarrow \infty}\left\|z^{n+1}\right\| /\left\|z^{n}\right\|=1$.

Proof. We have

$$
\begin{aligned}
\frac{\left\|z^{n+1}\right\|}{\left\|z^{n}\right\|} / \frac{\left\|z^{n}\right\|}{\left\|z^{n-1}\right\|} & =\frac{\left\|z^{n+1}\right\| \cdot\left\|z^{n-1}\right\|}{\left\|z^{n}\right\|^{2}}=\frac{\left(\int\left|z^{n+1}\right|^{2} d \sigma\right)^{1 / 2} \cdot\left(\int\left|z^{n-1}\right|^{2} d \sigma\right)^{1 / 2}}{\int\left|z^{n}\right|^{2} d \sigma} \\
& \geq \frac{\int\left|z^{n+1}\right|\left|z^{n-1}\right| d \sigma}{\int\left|z^{n}\right|^{2} d \sigma}=1 .
\end{aligned}
$$

Thus $\left\|z^{n+1}\right\| /\left\|z^{n}\right\|$ is increasing. Hence

$$
\liminf \frac{\left\|z^{n+1}\right\|}{\left\|z^{n}\right\|}=\limsup \frac{\left\|z^{n+1}\right\|}{\left\|z^{n}\right\|}=T .
$$

We first show that $T \geq 1$. Indeed, if $\limsup \left\|z^{n+1}\right\| /\left\|z^{n}\right\|<1$, then there exist $\delta>0$ and $N \in \mathbb{N}$ such that $T+\delta<1$ and $\left\|z^{n+1}\right\| /\left\|z^{n}\right\| \leq T+\delta$ for 
$n>N$. So,

$$
\begin{aligned}
\sqrt[n]{\left\|z^{n}\right\|} & =\sqrt[n]{\frac{\|z\|}{\|1\|} \cdots \frac{\left\|z^{N}\right\|}{\left\|z^{N-1}\right\|}} \cdot \sqrt[n]{\frac{\left\|z^{N+1}\right\|}{\left\|z^{N}\right\|} \cdots \frac{\left\|z^{n}\right\|}{\left\|z^{n-1}\right\|}} \\
& \leq \sqrt[n]{M} \cdot \sqrt[n]{(T+\delta)^{n-N}}
\end{aligned}
$$

Thus $\liminf \sqrt[n]{\left\|z^{n}\right\|} \leq T+\delta<1$. This contradicts Proposition 2.1.

Next we show that $T \leq 1$. Indeed, otherwise there exist $\beta>0$ and $N \in \mathbb{N}$ such that $\left\|z^{N+1}\right\| /\left\|z^{N}\right\|>1+\beta$ for $n>N$. So

$$
\begin{aligned}
\sqrt[n]{\left\|z^{n}\right\|} & =\sqrt[n]{\frac{\|z\|}{1} \cdots \frac{\left\|z^{N}\right\|}{\left\|z^{N-1}\right\|} \frac{\left\|z^{N+1}\right\|}{\left\|z^{N}\right\|} \cdots \frac{\left\|z^{n}\right\|}{\left\|z^{n-1}\right\|}} \\
& \geq \sqrt[n]{M} \cdot \sqrt[n]{(1+\beta)^{n-N}}
\end{aligned}
$$

This means that $\liminf \sqrt[n]{\left\|z^{n}\right\|} \geq 1+\beta$, contrary to Proposition 2.1 again. This completes the proof.

Since the reproducing kernel of $X$ is of the form

$$
K_{\lambda}(z)=\sum_{m=0}^{\infty} \frac{\bar{\lambda}^{m} z^{m}}{\left\|z^{m}\right\|^{2}}
$$

and $X$ is completely determined by $K_{\lambda}(z)$, it is interesting to know which spaces are ordered through $\|z\|^{n}$. We first give a proposition to illustrate which spaces are not ordered.

Proposition 2.5. Let $X$ be an analytic Hilbert space with a reproducing kernel $K_{\lambda}(z)=\sum_{n=0}^{\infty} z^{n} \bar{\lambda}^{n} /\|z\|^{2}$. If $\lim \inf \left\|z^{n+1}\right\| /\left\|z^{n}\right\|=0$, then $X$ is not ordered.

Proof. By the assumption, there exist $n_{k}>0$ such that $\left\|z^{n_{k}+1}\right\| /\left\|z^{n_{k}}\right\|<$ $1 / k^{2}$. Let $g(z)=\sum_{k=1}^{\infty} z^{n_{k}} /\left\|z^{n_{k}}\right\|$. Then $g \notin X$. But

$$
\|z g(z)\|^{2}=\sum_{k=0}^{\infty} \frac{\left\|z^{n_{k}+1}\right\|}{\left\|z^{n_{k}}\right\|}<\sum_{n=0}^{\infty} \frac{1}{k^{2}}<\infty .
$$

By Proposition 2.3, $X$ is not ordered.

Proposition 2.6. Let $X$ be an ordered analytic Hilbert space. Then there exists $M>0$ such that $\left\|z^{n}\right\| \leq M$ for all $n$.

Proof. Otherwise there exist $n_{k}>0$ such that $\left\|z^{n_{k}}\right\| \geq k$. Set $f(z)=$ $\sum_{k=1}^{\infty} z^{n_{k}} / k^{3 / 2}$. It is easy to see that $f \in H^{\infty}$. By Proposition 2.3, we have $f \in X$. On the other hand,

a contradiction.

$$
\|f\|^{2}=\sum_{k=1}^{\infty} \frac{\left\|z^{n_{k}}\right\|^{2}}{k^{3}} \geq \sum_{k=1}^{\infty} \frac{1}{k}
$$


By Proposition 2.6, the classical Dirichlet space is not ordered. Furthermore, if $\lim \inf \left\|z^{n+1}\right\| /\left\|z^{n}\right\|>1$, then $X$ is not ordered. If lim sup $\left\|z^{n+1}\right\| /\left\|z^{n}\right\|$ $<1$, then $X$ is not an analytic Hilbert space over $\mathbb{D}$. So when one studies which spaces are ordered, one only has to consider the following cases:

(1) $\lim \sup \left\|z^{n+1}\right\| /\left\|z^{n}\right\| \geq 1$,

(2) $0<\liminf \left\|z^{n+1}\right\| /\left\|z^{n}\right\| \leq 1$.

3. Some characterizations of analytic Hilbert spaces. In this section we first give a sufficient condition for an analytic Hilbert space to be ordered. Then we will give examples to show that each condition in our theorem is sharp to some extent. The following is one of the main results of this paper.

Theorem 3.1. Let $X$ be an analytic Hilbert space. If

(1) $\liminf \left\|z^{n+1}\right\| /\left\|z^{n}\right\|=1$;

(2) there exists $M>0$ such that $\left\|z^{n+k}\right\| /\left\|z^{n}\right\| \leq M$ for all $n, k>0$, then $X$ is ordered.

Proof. Let $\mathcal{M}$ denote the multiplication algebra of $X$. We first show that, under condition (2), $\mathcal{M}=H^{\infty}$. Note that $H^{\infty}$ is contained in the Hardy space $H^{2}$. Assume that $f(z)=\sum_{n=0}^{\infty} a_{n} z^{n} \in X$. Then $g(z)=$ $\sum_{n=0}^{\infty} a_{n}\left\|z^{n}\right\| z^{n} \in H^{2}$. Thus for each $b(z)=\sum_{n=0}^{\infty} b_{n} z^{n} \in H^{\infty}, b g \in H^{2}$, or equivalently,

$$
\sum_{k=0}^{\infty}\left|a_{0} b_{k}+a_{1} b_{k-1}\|z\|+a_{2} b_{k-2}\left\|z^{2}\right\|+\cdots+a_{k} b_{0}\left\|z^{k}\right\|\right|^{2}<\infty .
$$

It is obvious that $f(z)=\sum_{n=0}^{\infty} a_{n} z^{n} \in X$ implies that $\sum_{n=0}^{\infty}\left|a_{n}\right| z^{n} \in X$. If $b(z)=\sum_{n=0}^{\infty} b_{n} z^{n} \in H^{\infty}$, we will show that $\sum_{n=0}^{\infty}\left|b_{n}\right| z^{n} \in H^{\infty}$. Indeed, otherwise there exists a sequence $\left\{z_{n}\right\} \subset \mathbb{D}$ such that $\lim _{n \rightarrow \infty}\left|b_{n}\right| z_{n}=\infty$. Let $w_{n}=\left(\left|b_{n}\right| / b_{n}\right) \cdot z_{n}$. We have $\lim _{n \rightarrow \infty} b_{n} w_{n}=\infty$ and this contradicts $b \in H^{\infty}$.

So without loss of generality, we may assume that $a_{n}, b_{n} \geq 0$ for $n=$ $1,2, \ldots$ Since there exists $M>0$ such that $\left\|z^{n+k}\right\| /\left\|z^{n}\right\| \leq M$ for all $n, k>0$, we have

$$
\begin{aligned}
\|b f\|^{2} & =\sum_{k=0}^{\infty}\left|a_{0} b_{k}+a_{1} b_{k-1}+\cdots+a_{k} b_{0}\right|^{2}\left\|z^{k}\right\|^{2} \\
& =\sum_{k=0}^{\infty}\left|\frac{\left\|z^{k}\right\|}{\left\|z^{0}\right\|} a_{0} b_{k}\left\|z^{0}\right\|+\frac{\left\|z^{k}\right\|}{\left\|z^{1}\right\|} a_{1} b_{k-1}\left\|z^{1}\right\|+\cdots+\frac{\left\|z^{k}\right\|}{\left\|z^{k}\right\|} a_{k} b_{0}\left\|z^{k}\right\|\right|^{2} \\
& \leq M^{2} \sum_{n=0}^{\infty}\left|a_{0} b_{k}+a_{1}\|z\| b_{k-1}+a_{2}\left\|z^{2}\right\| b_{k-2}+\cdots+a_{k}\left\|z^{k}\right\| b_{0}\right|^{2}<\infty
\end{aligned}
$$

Thus $b f \in X$. So $H^{\infty} \subset \mathcal{M}$. 
The fact that $\mathcal{M} \subset H^{\infty}$ is well known for experts, we give a proof here just for convenience. In fact,

$$
\left|f(\lambda) K_{\lambda}(\lambda)\right|=\left|\left\langle M_{f} K_{\lambda}, K_{\lambda}\right\rangle\right| \leq\left\|M_{f}\right\| \cdot\left\|K_{\lambda}\right\|^{2}=\left\|M_{f}\right\| \cdot\left|K_{\lambda}(\lambda)\right|,
$$

and thus $|f(z)| \leq\left\|M_{f}\right\|$.

Now we only need to show that for each $|\alpha|<1$, under condition (1), $(z+\alpha) f \in X$ implies $f \in X$. For $f(z)=\sum_{n=0}^{\infty} a_{n} z^{n}$, we denote $\sum_{n=0}^{N} a_{n} z^{n}$ by $f_{N}(z)$.

If $\alpha=0$, since $\liminf _{n \rightarrow \infty}\left\|z^{n+1}\right\| /\left\|z^{n}\right\|=1$, there exists $N \in \mathbb{N}$ such that $\left\|z^{n+1}\right\|>\frac{1}{2}\left\|z^{n}\right\|$ for $n>N$. Then

$$
\begin{aligned}
\|f\|^{2} & =\sum_{n=0}^{\infty}\left|a_{n}\right|^{2}\left\|z^{n}\right\|^{2}=\sum_{n=0}^{N}\left|a_{n}\right|^{2}\left\|z^{n}\right\|^{2}+\sum_{n=N+1}^{\infty}\left|a_{n}\right|^{2}\left\|z^{n}\right\|^{2} \\
& \leq \sum_{n=0}^{N}\left|a_{n}\right|^{2}\left\|z^{n}\right\|^{2}+2 \sum_{n=N+1}^{\infty}\left|a_{n}\right|^{2}\left\|z^{n+1}\right\|^{2} \\
& \leq \sum_{n=0}^{N}\left|a_{n}\right|^{2}\left\|z^{n}\right\|^{2}+2\|z f\|^{2} .
\end{aligned}
$$

Thus $z f \in X$ implies $f \in X$.

In the case of $0<|\alpha|<1$, by the assumption $\liminf _{n \rightarrow \infty}\left\|z^{n+1}\right\| /\left\|z^{n}\right\|$ $=1$, we pick $\delta>0$ and $N_{\alpha} \in \mathbb{N}$ such that $\left\|z^{n+1}\right\|^{2} /\left\|z^{n}\right\|^{2} \geq \delta+|\alpha|$ for $n>N_{\alpha}$. Therefore for $N>N_{\alpha}$, we have

$$
\begin{aligned}
\left\|\alpha f_{N}\right\|^{2} & =\sum_{n=0}^{N}|\alpha|^{2}\left|a_{n}\right|^{2}\left\|z^{n}\right\|^{2} \\
& =\sum_{n=0}^{N_{\alpha}}|\alpha|^{2}\left|a_{n}\right|^{2}\left\|z^{n}\right\|^{2}+\sum_{n=N_{\alpha}+1}^{N}|\alpha|^{2}\left|a_{n}\right|^{2} \frac{\left\|z^{n}\right\|^{2}}{\left\|z^{n+1}\right\|^{2}}\left\|z^{n+1}\right\|^{2} \\
& \leq M_{\alpha}+\frac{|\alpha|^{2}}{(\delta+|\alpha|)^{2}}\left\|z f_{N}\right\|,
\end{aligned}
$$

where $M_{\alpha}=\sum_{n=0}^{N_{\alpha}}|\alpha|^{2}\left|a_{n}\right|^{2}\left\|z^{n}\right\|^{2}$. Thus

$$
\begin{aligned}
\left\|z f_{N}\right\| & \leq\left\|(z+\alpha) f_{N}\right\|+\left\|\alpha f_{N}\right\| \\
& \leq\left\|(z+\alpha) f_{N}\right\|+\sqrt{M_{\alpha}+\gamma\left\|z f_{N}\right\|^{2}},
\end{aligned}
$$

where $\gamma=|\alpha|^{2} /(\delta+|\alpha|)^{2}<1$. Since

$$
(z+\alpha) f_{N}(z)=\alpha a_{0}-\alpha a_{N+1} z^{N+1}+\sum_{n=1}^{N+1}\left(a_{n-1}+\alpha a_{n}\right) z^{n},
$$




$$
\begin{aligned}
(z+\alpha) f(z) & =\alpha a_{0}+\sum_{n=1}^{\infty}\left(a_{n-1}+\alpha a_{n}\right) z^{n} \\
{[(z+\alpha) f]_{N}(z) } & =\alpha a_{0}+\sum_{n=1}^{N}\left(a_{n-1}+\alpha a_{n}\right) z^{n}
\end{aligned}
$$

we have

$$
\begin{aligned}
\left\|(z+\alpha) f_{N}\right\| & \leq\left|\alpha a_{0}\right|+\left\|[(z+\alpha) f]_{N+1}\right\|+\left|\alpha a_{N+1}\right|\left\|z^{N+1}\right\| \\
& \leq\left|\alpha a_{0}\right|+\|(z+\alpha) f\|+\left|\alpha a_{N+1}\right|\left\|z^{N+1}\right\| .
\end{aligned}
$$

Next we will show that

$$
\liminf _{n \rightarrow \infty}\left|a_{n+1}\right|\left\|z^{n+1}\right\| \neq \infty
$$

Indeed, otherwise there exists $n_{0}>0$ such that $\left|a_{n}\right| \neq 0$ whenever $n>n_{0}$. Since $(z+\alpha) f(z)=\alpha a_{0}+\sum_{n=1}^{\infty}\left(a_{n-1}+\alpha a_{n}\right) z^{n} \in X$, there exists $n_{1}>0$ such that

$$
\left|a_{n-1}+\alpha a_{n}\right|\left\|z^{n}\right\|<1 \quad \text { for } n>n_{1} .
$$

So

$$
\left\|\alpha a_{n} z^{n}\right\|-1 \leq\left\|a_{n-1} z^{n}\right\| \leq\left\|\alpha a_{n} z^{n}\right\|+1
$$

whenever $n>n_{1}$. Hence

$$
\lim _{n \rightarrow \infty} \frac{\left|a_{n-1}\right|}{\left|a_{n}\right|}=|\alpha|<1 .
$$

This contradicts $f$ being analytic in the unit disk. Thus (3) holds. Therefore there exist a subsequence $\left\{n_{k}\right\}_{k=1}^{\infty}$ and $M>0$ such that

$$
\left\|a_{n_{k}} z^{n_{k}}\right\| \leq M \quad \text { for } k=1,2, \ldots
$$

If $z f \notin X$, then $\lim _{n \rightarrow \infty}\left\|z f_{n}\right\|=\|z f\|=\infty$. Consequently,

$$
\lim _{k \rightarrow \infty}\left\|z f_{n_{k}-1}\right\|=\infty \text {. }
$$

Combining (1), (2) and (5), we get

(6) $\left\|z f_{n_{k}-1}\right\| \leq\left|\alpha a_{0}\right|+\|(z+\alpha) f\|+\left|\alpha a_{n_{k}}\right|\left\|z^{n_{k}}\right\|+\sqrt{M_{\alpha}+\gamma\left\|z f_{n_{k}-1}\right\|^{2}}$.

Now divide (6) by $\left\|z f_{n_{k}-1}\right\|$ and let $k \rightarrow \infty$ to get $1 \leq \sqrt{\gamma}$. This is impossible. Thus we have $z f \in X$. So $f \in X$.

REMARK 3.1. Let $Y$ be an analytic Hilbert space whose inner product is induced by a positive measure. By definition, it is easy to see that $Y$ is ordered. So it is natural to ask whether one can determine that $Y$ is ordered by using Theorem 3.1. In fact, by Proposition 2.4 and the fact that $\left\|z^{n}\right\| \leq\left\|z^{n+k}\right\|$ for all positive integers $n$ and $k$, it is obvious that $Y$ is ordered. 
In the remainder of this section, we will give some examples to show that Theorem 3.1 is sharp to some extent. We recall that $\lim \sup \left\|z^{n+1}\right\| /\left\|z^{n}\right\| \geq 1$ is necessary for $X$ to be an analytic Hilbert space. So all the examples in the following satisfy this condition.

The first example is an analytic Hilbert space which is not ordered and satisfies $\lim \inf \left\|z^{n+1}\right\| /\left\|z^{n}\right\|<1$.

EXAMPLE 3.1. Let $X$ be an analytic Hilbert space with

$$
\left\|z^{n}\right\|= \begin{cases}1, & 0 \leq n \leq 11, \\ \frac{1}{k}, & 2^{k-1}+k \leq n \leq 2^{k}-k \text { for } k \geq 4, \\ \frac{1}{k 2^{j}}, & n=2^{k}-k+j, 0<j \leq k, \\ \frac{1}{k 2^{k-i}}, & n=2^{k}+i, 0<i<k .\end{cases}
$$

Then $\lim \sup \left\|z^{n+1}\right\| /\left\|z^{n}\right\|=2$, $\liminf \left\|z^{n+1}\right\| /\left\|z^{n}\right\|=1 / 2$ and $X$ is not ordered.

Proof. Let

$$
b_{k}(z)=z^{2^{k}-k}+2 z^{2^{k}-k+1}+2^{2} z^{2^{k}-k+2}+\cdots+2^{k-1} z^{2^{k}-k+(k-1)}+2^{k} z^{2^{k}}
$$

and $f(z)=\sum_{k=4}^{\infty} b_{k}(z)$. Obviously, if we write $f(z)=\sum_{n=0}^{\infty} a_{n} z^{n}$, then $\left|a_{n}\right| \leq n$. Thus $f \in \operatorname{Hol}(\mathbb{D})$. Since

$$
\|f\|^{2}=\sum_{k=4}^{\infty}\left\|b_{k}\right\|^{2}=\sum_{k=4}^{\infty} \frac{k+1}{k^{2}}=\infty,
$$

we have $f \notin X$. But

$$
\left(z-\frac{1}{2}\right) f(z)=\sum_{k=4}^{\infty}\left(z-\frac{1}{2}\right) b_{k}(z)=\sum_{k=4}^{\infty}\left(-\frac{1}{2} z^{2^{k}-k}+2^{k} z^{2^{k}+1}\right)
$$

So

$$
\left\|\left(z-\frac{1}{2}\right) f(z)\right\|^{2}=\sum_{k=4}^{\infty}\left(\frac{1}{(2 k)^{2}}+\left(\frac{2}{k}\right)^{2}\right)<\infty
$$

and $(z-1 / 2) f(z) \in X$. By Proposition 2.3, $X$ is not ordered.

The next example exhibits an ordered analytic Hilbert space such that $\liminf \left\|z^{n+1}\right\| /\left\|z^{n}\right\|<1$.

EXAmPLE 3.2. Let $X$ be an analytic Hilbert space with $\left\|z^{2 k}\right\|=1$ and $\left\|z^{2 k+1}\right\|=2$ for $k=0,1, \ldots$ Then $\lim \sup \frac{\left\|z^{n+1}\right\|}{\left\|z^{n}\right\|}=2, \liminf \frac{\left\|z^{n+1}\right\|}{\left\|z^{n}\right\|}=1 / 2$ and $X$ is ordered. 
Proof. Since $\left\|z^{n+k}\right\| /\left\|z^{n}\right\|$ is uniformly bounded, by Theorem 3.1 we have $\mathcal{M}_{X}=H^{\infty}$. So we only need to show that $(z+\alpha) f(z) \in X$ implies $f \in X$ for each $\alpha \in \mathbb{D}$.

Set $f(z)=\sum_{n=0}^{\infty} a_{n} z^{n}$. Then

$$
\begin{aligned}
\|z f\|^{2} & =\sum_{n=0}^{\infty}\left|a_{n}\right|^{2}\left\|z^{n+1}\right\|^{2}=\sum_{k=0}^{\infty}\left|a_{2 k}\right|^{2}\left\|z^{2 k+1}\right\|^{2}+\sum_{k=0}^{\infty}\left|a_{2 k+1}\right|^{2}\left\|z^{2 k+2}\right\|^{2} \\
& =4 \sum_{k=0}^{\infty}\left|a_{2 k}\right|^{2}\left\|z^{2 k}\right\|^{2}+\frac{1}{4} \sum_{k=0}^{\infty}\left|a_{2 k+1}\right|^{2}\left\|z^{2 k+1}\right\|^{2} \geq \frac{1}{4} \sum_{n=0}^{\infty}\left|a_{n}\right|^{2}\left\|z^{2 n}\right\|^{2} .
\end{aligned}
$$

Thus $z f \in X$ implies $f \in X$.

Assume that $(z+\alpha) f \in X$ for $0<|\alpha|<1$ and

$$
(z+\alpha) f(z)=\alpha a_{0}+\sum_{n=1}^{\infty}\left(\alpha a_{n}+a_{n-1}\right) z^{n} .
$$

Let

$$
f_{1}(z)=\alpha a_{0}+\sum_{k=1}^{\infty}\left(\alpha a_{2 k}+a_{2 k-1}\right) z^{2 k}, \quad f_{2}(z)=\sum_{k=1}^{\infty}\left(\alpha a_{2 k+1}+a_{2 k}\right) z^{2 k+1} .
$$

Set

$$
f_{0}(z)=\frac{f_{2}(z)}{z}=\sum_{k=1}^{\infty}\left(\alpha a_{2 k+1}+a_{2 k}\right) z^{2 k} .
$$

Since $(z+\alpha) f(z) \in X$, it follows that $f_{1}, f_{2} \in X$. Since $z f_{0}(z)=f_{2}(z) \in X$, we have $f_{0} \in X$ and

$$
\left(f_{2}-\alpha f_{0}\right)(z)=-\alpha^{2} a_{1}+\sum_{k=1}^{\infty}\left(a_{2 k-1}-\alpha^{2} a_{2 k+1}\right) z^{2 k} \in X .
$$

So

$$
\sum_{k=1}^{\infty}\left|a_{2 k-1}-\alpha^{2} a_{2 k+1}\right|^{2}\left\|z^{2 k}\right\|^{2}<\infty
$$

We also note that Theorem 3.1 in fact shows the following:

Let $\alpha \in \mathbb{D}$ and $g(z)=\sum_{n=0}^{\infty} b_{n} z^{n}$ be analytic in $\mathbb{D}$ and $\liminf _{n \rightarrow \infty} r_{n+1} / r_{n}$ $=1$ where $r_{n}>0$. Then $\sum_{n=0}^{\infty}\left(b_{n}+\alpha b_{n+1}\right)^{2} r_{n}^{2}<\infty$ implies $\sum_{n=0}^{\infty} b_{n}^{2} r_{n}^{2}<\infty$.

Let $a_{2 k-1}=b_{k}$ and $r_{k}=\left\|z^{2 k}\right\|=1$. Then (7) is equivalent to

$$
\sum_{k=0}^{\infty}\left|b_{k}-\alpha^{2} b_{k+1}\right|^{2} r_{k}^{2}<\infty
$$

This implies that

$$
\sum_{k=1}^{\infty}\left|b_{k}\right|^{2} r_{k}^{2}<\infty
$$


or equivalently

$$
\sum_{k=1}^{\infty}\left|a_{2 k-1}\right|^{2}\left\|z^{2 k}\right\|^{2}<\infty
$$

Since $\left\|z^{2 k+1}\right\|=2\left\|z^{2 k}\right\|$, we have

$$
\sum_{k=1}^{\infty}\left|a_{2 k-1}\right|^{2}\left\|z^{2 k-1}\right\|^{2}<\infty
$$

Thus

$$
h(z)=\sum_{k=1}^{\infty} a_{2 k-1} z^{2 k-1}=\sum_{k=0}^{\infty} a_{2 k+1} z^{2 k+1} \in X .
$$

We also have $f_{2}(z)-\alpha h(z)=\sum_{k=0}^{\infty} a_{2 k} z^{2 k+1} \in X$.

By using $\left\|z^{2 k+1}\right\|=2\left\|z^{2 k}\right\|$ again, we get $h_{1}(z)=\sum_{k=0}^{\infty} a_{2 k} z^{2 k} \in X$. Thus $f=h_{1}+h \in X$.

Examples 3.1 and 3.2 show that condition (1) of Theorem 3.1 is sharp to some extent. The following example shows the necessity of condition (2) of Theorem 3.1.

Example 3.3. Let $X$ be an analytic Hilbert space and $\left\{\left\|z^{n}\right\|\right\}_{n=1}^{\infty}$ be the following sequence:

1 ,

$$
\begin{aligned}
& 1-\frac{1}{2^{4}}, \quad 1-\frac{2}{2^{4}}, \quad \ldots, \quad \frac{1}{2^{3}}, \quad \frac{1}{2^{3}}, \quad 1, \\
& 1-\frac{1}{3^{4}}, \quad 1-\frac{2}{3^{4}}, \quad \ldots, \quad \frac{1}{3^{3}}, \quad \frac{1}{3^{3}}, \quad \frac{2^{3}}{3^{3}}, \quad 1,
\end{aligned}
$$$$
1-\frac{1}{k^{4}}, \quad 1-\frac{2}{k^{4}}, \quad \ldots, \quad \frac{1}{k^{3}}, \quad \frac{1}{k^{3}}, \quad\left(\frac{2}{k}\right)^{3}, \quad \ldots, \quad\left(\frac{k-1}{k}\right)^{3}, \quad 1,
$$

Set $m_{k}=\sum_{i=1}^{k} i^{4}$. Then $\left\|z^{m_{k}}\right\|=1,\left\|z^{m_{k}-k}\right\|=1 / k^{3},\left\|z^{m_{k}}\right\| /\left\|z^{m_{k}-k}\right\|=k^{3}$ and $X$ is not ordered.

Proof. We first note that the number of elements in the set

$$
\left\{1-\frac{1}{k^{4}}, 1-\frac{2}{k^{4}}, \ldots, \frac{1}{k^{3}}, \frac{1}{k^{3}},\left(\frac{2}{k}\right)^{3}, \ldots,\left(\frac{k-1}{k}\right)^{3}, 1\right\}
$$

is $k^{4}$, because $\frac{1-1 / k^{3}}{1 / k^{4}}+k=k^{4}$.

Since $\lim _{n \rightarrow \infty}\left\|z^{n+1}\right\| /\left\|z^{n}\right\|=1$, condition (1) of Theorem 2.1 is satisfied. Next we will show that $H^{\infty} \nsubseteq \mathcal{M}_{X}$. In fact, take $a_{m_{k}}=1 / k, a_{m_{k}-k}=k^{2}$ and 
$a_{i}=0$ for $i \neq m_{k}, m_{k}-k$. Then $f(z)=\sum a_{n} z^{n} \in X$. Set $b(z)=\sum_{k=1}^{\infty} z^{k} / k^{2}$. It is obvious that $b \in H^{\infty}$. In this case,

$$
\begin{aligned}
\|b f\|^{2} & =\sum_{n=0}^{\infty}\left|a_{n} b_{0}+a_{k-1} b_{1}+\cdots+a_{0} b_{n}\right|^{2}\left\|z^{k}\right\|^{2} \\
& \geq \sum_{k=1}^{\infty}\left|a_{m_{k}} b_{0}+\cdots+a_{0} b_{m_{k}}\right|^{2}\left\|z^{m_{k}}\right\| \geq \sum_{k=1}^{\infty}\left|a_{m_{k}-k} b_{k}\right|^{2}=\infty .
\end{aligned}
$$

Thus $b f \notin X$.

4. Similarity of subspaces to $X$. The classical Beurling theorem states that all the invariant subspaces of the Hardy space are similar (unitarily equivalent) [Beu]. However, the situation in the Bergman space is quite different: two invariant subspaces are unitarily equivalent if and only if they are equal [Ri2]. The classification of the Bergman space (under similarity) is far from success [Bou, HKZ1, Zhu1, Zhu2]. In this section, we mainly study the similarity of invariant subspaces of ordered analytic Hilbert spaces. We prove that every finite-dimensional invariant subspace is similar to $X$ when $X$ is ordered. We also give examples to show that this may not be true when $X$ is not ordered. We first give a lemma.

Lemma 4.1. Let $X$ be an analytic Hilbert space and $|\alpha|<1$. Then $(z+\alpha) f \in X$ implies $f \in X$ if and only if $M_{z+\alpha}$ is bounded below.

Proof. " $\Rightarrow$ " First we show that the range of $T=M_{z+\alpha}$ is closed. In fact, if $(z+\alpha) f_{n}$ converges to $g$, then there exists $g_{0} \in \operatorname{Hol}(\mathbb{D})$ such that $g=(z+\alpha) g_{0}$. By the assumption, we have $g_{0} \in X$. Thus $R(T)=\{(z+\alpha) f \mid$ $f \in X,(z+\alpha) f \in X\}$ is closed.

Next we will show that $G(T)$, the graph of $T$, is closed. That is, for $f_{n} \stackrel{\|\|}{\longrightarrow} f$ and $(z+\alpha) f_{n} \stackrel{\|\|}{\rightarrow} g \in X$, we need to show that $f \in D(T)$ and $(z+\alpha) f=g$. Since $(z+\alpha) f_{n} \stackrel{\|\|}{\rightarrow} g$, there exists $g_{0} \in \operatorname{Hol}(\mathbb{D})$ such that $g=(z+\alpha) g_{0}$. Combining this with the assumption gives $g_{0} \in X$. Since $X$ is an analytic Hilbert space, $(z+\alpha) f_{n} \stackrel{\|\|}{\rightarrow}(z+\alpha) g_{0}$ implies $(z+\alpha) f_{n} \rightarrow$ $(z+\alpha) g_{0}$ pointwise. Thus $f_{n} \rightarrow g_{0}$ pointwise. Since $f_{n} \stackrel{\|\|}{\rightarrow} f$, we have $f=g_{0}$. Therefore, the graph of $T$ is closed and the inverse of $T$ is bounded.

" $\Leftarrow$ " If $M_{z+\alpha}$ is bounded below, then $R\left(M_{z+\alpha}\right)$ is closed. Thus

$$
M_{z+\alpha}^{-1}: R\left(M_{z+\alpha}\right) \rightarrow X
$$

is bounded. If $(z+\alpha) f=g \in X$ and $M_{z+\alpha}^{-1} g=h$, then

$$
M_{z+\alpha}(f-h)=(z+\alpha) f-M_{z+\alpha} M_{z+\alpha}^{-1} g=g-g=0 .
$$

Since both $f$ and $h$ are analytic in $\mathbb{D}$, we have $f=h$. So $f \in X$. 
To prove Theorem 4.1, we need a proposition due to Bourdon [Bou].

Lemma 4.2 ([Bou]). If $S: H \rightarrow H$ is bounded below and commutes with $T$, then $\operatorname{Ran} S$ is invariant for $T$ and is similar to $H$.

Let $\phi \in \operatorname{Hol}(\mathbb{D})$. We say that $\phi$ is a universal divisor of $X$ if for each $f \in X, f / \phi$ analytic in $\mathbb{D}$ implies $f / \phi \in X$. Recall that in the Hardy space each Blaschke product is a universal divisor and in the Bergman space, every finite product of interpolating Blaschke products is a universal divisor. When $X$ is an ordered analytic Hilbert space, each polynomial $p$ is a universal divisor.

Theorem 4.1. Let $X$ be an analytic Hilbert space and $N$ be an invariant subspace of $X$. Then the following are equivalent:

(1) $N$ is similar to $X$;

(2) $N$ is generated by $\phi \in X$ and $M_{\phi}$ is bounded below;

(3) $\phi$ is a universal divisor.

Proof. (1) $\Rightarrow(2)$. Assume that $A: X \rightarrow N$ is a similarity. Then $N=$ $[A(1)]$, where $[A(1)]$ denotes the closed subspace generated by $A(1)$. Since $A(z f)=z A(f)$ for all $f \in X$, there exists $\phi \in H^{\infty}$ such that $A=M_{\phi}$. Since $M_{\phi}$ is injective, $M_{\phi}: X \rightarrow N$ is bijective. Thus $A^{-1}=M_{\phi}^{-1}$ is bounded. For each polynomial $p$,

$$
\|p\|=\left\|M_{\phi}^{-1} M_{\phi} p\right\| \leq\left\|M_{\phi}^{-1}\right\|\left\|M_{\phi} p\right\| .
$$

Thus $\left\|M_{\phi} p\right\| \geq\left(1 /\left\|M_{\phi}^{-1}\right\|\right)\|p\|$ and $M_{\phi}$ is bounded below.

$(2) \Rightarrow(1)$. A direct application of Lemma 4.2.

$(2) \Rightarrow(3)$. If $M_{\phi}$ is bounded below, we want to show that for each $f \in$ $\operatorname{Hol}(\mathbb{D}), \phi f \in X$ implies $f \in X$. Since $M_{\phi}$ is bounded below, the range of $M_{\phi}$ is closed. Let $M_{\phi} f=g \in X$. Then

$$
M_{\phi}^{-1}: \operatorname{Ran} M_{\phi} \rightarrow X
$$

is bounded. We denote $M_{\phi}^{-1} g$ by $h$. It is obvious that $h \in X$. Note that $M_{\phi}(f-h)=\phi f-M_{\phi} M_{\phi}^{-1} g=g-g=0$ and both $f$ and $h$ are analytic in $\mathbb{D}$. So $f=g \in X$.

$(3) \Rightarrow(2)$. The proof is similar to that in [Hor]. We give it just for convenience. Note that the space

$$
\{f \in X \mid f / \phi \in \operatorname{Hol}(\mathbb{D})\}
$$

is closed in $X$. By the closed graph theorem, if $f$ is a universal divisor for $X$, then the map of $\{f \in X \mid f / \phi \in \operatorname{Hol}(\mathbb{D})\}$ into $X$ which sends $f \in X$ to $\phi f$ must be bounded. Equivalently, the map which sends $f \in X$ to $f \phi$ must be bounded below. 
Although Theorem 4.1 gives a necessary and sufficient condition for a subspace $M$ to be similar to $X$, it does not provide much information about $\phi$. In the case of the Hardy space, it is well known that $\phi$ can be chosen to be an inner function. In the case of the Bergman space, it is known [Bou, Zhu2] that $\phi$ is a finite product of interpolating Blaschke products.

If $X$ is ordered, then $\mathcal{M}=H^{\infty}$. Thus each finite-codimensional subspace of an ordered analytic Hilbert space is generated by a polynomial with all roots in $\mathbb{D}$ (Theorem 2.5 of $[\mathrm{AS}]$ ). By Proposition 2.3 and Lemma 4.1, we have the following:

Corollary 4.1. Let $X$ be ordered and $M$ be a finite-codimensional invariant subspace. Then $M$ is similar to $X$.

Corollary 4.1 fails to hold for analytic Hilbert spaces without order, as can be seen from the following example:

EXAMPLE 4.1. Let $Y$ be an analytic Hilbert space with $\|1\|=1,\left\|z^{2 k}\right\|=$ $2 k$ and $\left\|z^{2 k+1}\right\|=1 /(2 k+1)$ for $k=1,2, \ldots$ Let $[z]$ denote the invariant subspace generated by the ideal $z \mathcal{C}$. Then $[z]$ is not similar to $Y$.

In fact, if $[z]$ is similar to $X$, then $M_{z}$ is bounded below. This contradicts the argument in Example 2.1.

\section{References}

[AS] A. Abdollahi and K. Seddighi, Finite codimensional invariant subspaces of Banach spaces of analytic functions, Rocky Mountain J. Math. 29 (1999), 369-381.

[Beu] A. Beurling, On two problems concerning linear transformations in Hilbert spaces, Acta Math. 81 (1949), 239-255.

[Bou] P. Bourdon, Similarity of parts to the whole for certain multiplication operators, Proc. Amer. Math. Soc. 99 (1987), 563-567.

[CG] X. M. Chen and K. Y. Guo, Analytic Hilbert Modules, Res. Notes Math. 433, Chapman \& Hall/CRC, 2003.

[CGH] X. M. Chen, K. Y. Guo and S. Z. Hou, Analytic Hilbert spaces over complex plane, J. Math. Anal. Appl. 268 (2002), 684-700.

[DP] R. G. Douglas and V. I. Paulsen, Hilbert Modules over Function Algebras, Pitman Res. Notes in Math. 217, Longman, 1989.

[GH] K. Y. Guo and S. Z. Hou, Quasi-invariant subspaces generated by polynomials with nonzero leading terms, Studia Math. 164 (2004), 231-241.

[HKZ1] H. Hedenmalm, B. Korenblum and K. Zhu, Beurling type invariant subspaces of the Bergman spaces, J. London Math. Soc. (2) 53 (1996), 601-614.

[HKZ2] - , - - , Theory of Bergman Spaces, Grad. Texts in Math. 199, Springer, 2000.

[Hor] C. Horowitz, Factorization theorems for functions in the Bergman spaces, Duke Math. J. 44 (1977), 201-213.

[Ri1] S. Richter, Invariant subspaces in Banach spaces of analytic function, Trans. Amer. Math. Soc. 304 (1987), 585-616.

[Ri2] -, Unitary equivalence of invariant subspaces of Bergman and Dirichlet spaces, Pacific J. Math. 133 (1988), 151-156. 
[Zhu1] K. H. Zhu, Restriction of the Bergman shift to an invariant subspace, Quart. J. Math. Oxford (2) 48 (1997), 519-532.

[Zhu2] - Similarity of invariant subspaces of the Bergman space, preprint.

Department of Mathematics

Suzhou University

Suzhou, Jiangsu, 215006, P.R. China

E-mail: shou@suda.edu.cn

swei@suda.edu.cn

Received September 4, 2005

Revised version January 5, 2008 